\title{
RECONFIGURACIÓN DE REDES ELÉCTRICAS DE MEDIA TENSIÓN BASADA EN EL ALGORITMO DE PRIM
}

\section{RECONFIGURATION OF MEDIUM VOLTAGE NETWORKS BASED ON PRIM'S ALGORITHM}

\author{
Angely Cárcamo-Gallardo ${ }^{1} \quad$ Luis García Santander ${ }^{2} \quad$ Jorge E. Pezoa $^{2}$ \\ Recibido 10 de abril de 2006, aceptado 2 de marzo de 2007 \\ Received: April 10, 2006 Accepted: March 2, 2007
}

\begin{abstract}
RESUMEN
En este trabajo se presenta un nuevo algoritmo que permite reconfigurar un sistema de distribución (SD) de energía eléctrica minimizando la energía no suministrada (ENS). El SD se modela utilizando teoría de grafos, mientras que la ENS se formula recursivamente y se parametriza en términos de los índices de confiabilidad del SD. Empleando esta modelación se transforma el problema de optimización en el problema de encontrar el árbol de mínima expansión (AME) a partir del grafo que modela al SD, donde la métrica de distancia utilizada corresponde a la ENS a cada nodo del SD. Para encontrar de manera eficiente el AME se utiliza el algoritmo de Prim, ya que pertenece a la clase de algoritmos voraces en el cálculo del AME. Adicionalmente, se propone un algoritmo que realiza una revisión del AME obtenido analizando las topologías que fueron descartadas aleatoriamente durante el proceso de decisión. El desempeño del algoritmo de optimización se evalúa en sistemas de pruebas y en dos sistemas eléctricos reales.
\end{abstract}

Palabras clave: Algoritmo de Prim, reconfiguración, energía no suministrada, métodos de optimización, distribución de potencia.

\section{ABSTRACT}

This paper presents a novel algorithm to reconfigure an electric power distribution network (EPDN) minimizing its non-supplied energy (NSE). The EPDN is modeled using graph theory and the NSE is recursively formulated in terms of the reliability parameters of the EPDN. Based on this mathematical model, we transform the original optimization problem into the graph theory problem of finding the minimum spanning tree (MST) of a given graph, which models the EPDN. The distance metric employed by the searching algorithm is the NSE. In order to efficiently find the MST, Prim's algorithm is employed due to is greedy search behavior. In addition, a backtracking algorithm is used to check the MST obtained. The backtracking algorithm analyzes all the candidate topologies that were randomly discarded during the decision process. The performance of the optimization algorithm is evaluated by using testing systems and two actual EPDNS.

Keywords: Prim's algorithm, reconfiguration, non-supplied energy, optimization methods, power distribution.

\section{INTRODUCCIÓN}

Los sistemas de suministro eléctrico son los encargados de generar, transmitir y distribuir la energía eléctrica desde los centros de generación a los consumidores finales, por lo tanto, cualquier falla en una de sus etapas genera pérdidas importantes tanto para las empresas del rubro como para los clientes.
Hoy en día la calidad del servicio eléctrico de los sistemas de distribución (SD) de media tensión (MT) están siendo fiscalizado de manera más rigurosa gracias a una nueva ley de calidad de suministro eléctrico [1]. Una falla en un SD modifica severamente los índices de calidad de la empresa distribuidora, generando con ello importantes pérdidas económicas. Encontrar la red de MT que opere en forma radial usando los elementos más confiables hará que disminuya su probabilidad de falla, lo que implica

\footnotetext{
1 Department of Economics, the University of New Mexico, Albuquerque, NM, USA. acarcamo@unm.edu

2 Departamento de Ingeniería Eléctrica, Universidad de Concepción, Barrio Universitario s/n, Concepción, Chile. luigarci, jpezoa@udec.cl
} 
mejores índices de calidad para el SD. Es importante notar que la complejidad asociada a la búsqueda de dicha red no es menor, puesto que normalmente una red de distribución (RD) conecta cientos de cargas, lo que hace que un análisis combinatorio no sea viable. Además, el problema presenta restricciones adicionales: la RD en su operación no puede ser enmallada; todos los clientes deben permanecer conectados al sistema; las capacidades de las fuentes y de los conductores no deben ser sobrepasadas; y las tensiones de los nodos deben satisfacer los rangos de regulación de tensión establecidos por la legislación vigente.

La reconfiguración es el proceso de transformación de la topología de una RD de forma tal de obtener una configuración óptima que considere tanto la continuidad del servicio a los clientes como la reducción de posibles pérdidas económicas por parte de la empresa distribuidora. El proceso de optimización debe considerar parámetros asociados a la topología de la RD, su confiabilidad y los costos y beneficios que se generan al modificar sectores o la totalidad de la red.

El problema de la reconfiguración de una RD ha sido abordado utilizando diversos métodos y funcionales de optimización. Si clasificamos los trabajos de acuerdo al funcional empleado encontramos esencialmente dos tipos: (i) minimización de pérdidas debido al efecto Joule en las líneas de distribución, y (ii) optimización de otros funcionales relacionados con confiabilidad y tiempos de restauración del servicio. Dentro del primer grupo encontramos varios métodos heurísticos: McDecmott et al. utilizan un funcional de costo no lineal que minimiza las pérdidas eléctricas y un algoritmo de revisión una vez encontrado un mínimo local [1]; Goswami y Basu presentan un algoritmo para minimizar las pérdidas totales calculando en cada etapa los flujos de potencia [2], mientras que Gomes et al. emplean un algoritmo que calcula el flujo de carga y toma decisiones de reconfiguración minimizando las pérdidas totales del sistema [3]. Shirmohammadi y Hong presentan un método que considera inicialmente la red completamente conectada y los interruptores son abiertos selectivamente hasta eliminar cualquier ciclo en la red y obtener una configuración radial de mínimas pérdidas en las líneas [4]. Toune et al. presentan un excelente resumen teórico sobre métodos heurísticos aplicados al problema de restauración de servicio [5]. Por su parte, López et al. han utilizado la programación dinámica para minimizar las pérdidas de una RD [6], [7]. Min y Chan proponen en [8] un conjunto de índices asociados a grupos de interruptores y un algoritmo de cálculo que resulta muy eficiente y numéricamente muy preciso, cuando minimiza las pérdidas en las líneas y el tiempo de restauración del servicio.
Dentro del segundo grupo se encuentra el trabajo de Vega et al. [9], quienes estudian la confiabilidad de una red eléctrica industrial utilizando la energía no suministrada (ENS) como funcional de optimización. Popovic y Ciric [10] proponen un algoritmo jerárquico multiobjetivo que reduce la complejidad del problema, y a la vez, permite emplear múltiples funcionales que consideran pérdidas eléctricas, índices de confiabilidad y la ENS a las cargas. Posteriormente, el mismo grupo introduce un algoritmo basado en lógica difusa y teoría de probabilidades que les permite minimizar la ENS promedio [11]. Huang propone el uso de una red difusa de causa-efecto y la transformación, por medio de factores ponderadores, de un proceso de optimización multiobjetivo en un funcional simple que minimiza el tiempo de restauración del servicio [12]. Recientemente, Rodríguez y Vargas han propuesto un nuevo método heurístico que también usa lógica difusa y minimiza el tiempo de restauración del servicio [13].

En este trabajo se formula y presenta un algoritmo de reconfiguración de RD que minimiza la ENS del sistema. Empleando teoría de grafos, se obtiene un modelo matemático para la RD y se formula, recursivamente, la ENS en términos de los parámetros de confiabilidad del sistema. Con esto se procede a transformar el problema de minimización de la ENS en el problema de teoría de grafos de la obtención del árbol de mínima expansión (AME) a partir de un cierto grafo dado. El AME se obtiene mediante la implementación del algoritmo de Prim, el que emplea la ENS como métrica para la distancia entre los nodos del SD. La principal ventaja de utilizar el algoritmo de Prim para encontrar el AME es que su tiempo de ejecución es de orden logarítmico [14-16]. Además, el algoritmo propuesto considera una etapa de revisión de la topología radial resultante, la que consiste en evaluar aquellas conexiones descartadas aleatoriamente durante el proceso de decisión. Se debe recordar que durante la ejecución del algoritmo de Prim, en ocasiones, más de una conexión puede proporcionar una ruta óptima, por lo que aleatoriamente debe seleccionarse una ruta y descartar las demás. Esta etapa de revisión permite mitigar la naturaleza voraz del algoritmo de Prim y los efectos de la variación en la ENS del sistema cuando se agrega un nuevo nodo al árbol en evaluación. El desempeño del algoritmo se evaluó utilizando un sistema de pruebas sencillo y luego se procedió a evaluar dos sistemas reales considerablemente más complejos.

\section{FUNDAMENTACIÓN TEÓRICA}

En esta sección se presentan los aspectos teóricos asociados al problema. Primeramente se define la ENS 
asociada a una RD y se establece su dependencia con la topología del sistema. A continuación se desarrolla la formulación matemática del problema utilizando teoría de grafos y se define formalmente el algoritmo de búsqueda propuesto.

\section{La energía no suministrada de un sistema de distribución}

Considere un SD de $n$ nodos, $n>1$, donde el nodo 1 se define como el nodo fuente, es decir, aquel nodo que suministra la energía eléctrica. Considere también que el $i$-ésimo nodo, $i \in\{1,2, \ldots, n\}$, consume una cierta potencia activa $P_{i}$. Considere también que cada línea de distribución de energía tiene dos parámetros de confiabilidad asociados a ella: la indisponibilidad asociada al tiempo de reparación de la línea y la indisponibilidad asociada al tiempo de restauración del servicio. Se define la indisponibilidad asociada al tiempo de reparación de la línea como $U_{\{l, m\}}=\lambda_{\{l, m\}} t_{\{l, m\}}^{(r e p)}, l, m \in\{1,2, \ldots, n\}$, donde $\lambda_{\{l, m\}}$ es la tasa de falla por año de la línea que distribuye la energía entre los nodos $l$ y $m$, mientras que $t_{\{l, m\}}^{(r e p)}$ es el tiempo de reparación, en horas, de dicha línea. Se define también la indisponibilidad asociada al tiempo de restauración del servicio como $U_{\{l, m\}}^{\prime}=\lambda_{\{l, m\}} t_{\{l, m\}}^{(\text {rest })}$, donde $t_{\{l, m\}}^{(\text {rest })}$ es el tiempo de restauración del servicio, en horas, en la línea que une los nodos $l$ y $m$. Además, se asume también que todas las líneas de distribución poseen un interruptor, lo que permite abrir o reconfigurar la $\mathrm{RD}$ en cualquier punto del sistema. Finalmente, y para simplificar el análisis, se define que $P_{1}=0$, pues corresponde a la fuente de energía, y $U_{\{l, l\}}=U_{\{l, l\}}^{\prime}=0$.

La ENS por el SD a un nodo se define como el producto entre la indisponibilidad asociada al nodo y su potencia activa. La indisponibilidad de un nodo se define como la suma de las indisponibilidades asociadas al tiempo de reparación de todas aquellas líneas que conectan directamente el nodo en análisis al nodo fuente, más la indisponibilidad asociada al tiempo de restauración de todas aquellas líneas que: (i) están aguas arriba del nodo pero no pertenecen al enlace directo entre el nodo fuente y el nodo evaluado, y (ii) están aguas abajo del nodo en evaluación. Así, se define la ENS de un SD como la suma de la ENS a cada uno de los nodos que pertenecen al sistema.

Por ejemplo, considere que el SD de la figura 1 está formado inicialmente sólo por las tres líneas de trazo continuo, la ENS al nodo 2 se calcula como:

$$
E N S_{2}=\left(U_{\{1,2\}}+U_{\{2,4\}}^{\prime}\right) P_{2}
$$

Notar que la línea $\{1,3\}$ no se considera en el cálculo de la ENS, pues cada nueva rama generada a partir del nodo fuente es un circuito independiente de energía eléctrica. Si se considera ahora que el SD tiene las cuatro líneas conectadas, entonces la ENS del nodo 2 se calcula como:

$$
E N S_{2}=\left(U_{\{1,2\}}+U_{\{2,4\}}^{\prime}+U_{\{2,5\}}^{\prime}\right) P_{2}
$$

A través de este ejemplo es fácil notar que la ENS a un nodo depende de la topología del SD, y por lo tanto, la ENS del SD es también dependiente de la topología de la red.

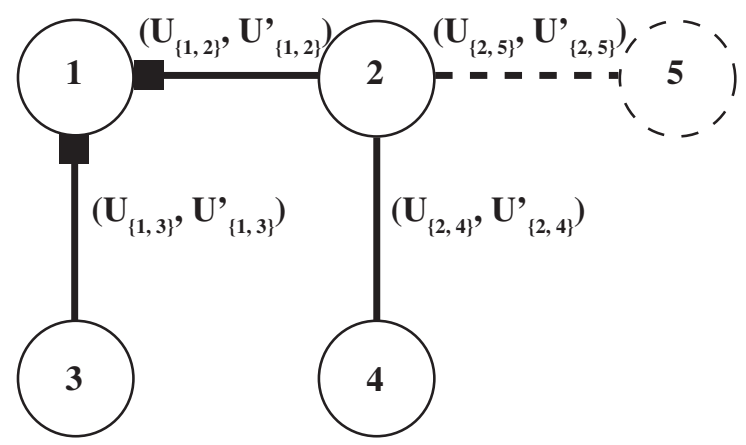

Figura 1. Un SD formado inicialmente por 4 nodos, al que se le agrega posteriormente el nodo 5. Notar que producto de la modificación de la topología del SD la ENS de éste se modifica.

\section{Modelación del sistema utilizando teoría de grafos}

Utilizando notación de teoría de grafos [15], se puede representar el SD por medio del grafo no orientado $G_{n}=\left(N_{n}, L_{n}\right)$, donde $N_{n}=\{1,2, \ldots, n\}$ es el conjunto de vértices del grafo, cuya cardinalidad es $\left|N_{n}\right|=n, \mathrm{y} L_{n}=\{\{1,2\},\{2,3\}, \ldots,\{n-1, n\}\}$ es el conjunto de aristas de $G_{n}$, de cardinalidad $\left|L_{n}\right|=p \geq n-1$. Sean: $A_{j}(n)=\left(N_{n}, L_{A_{j}}\right)$ el j-ésimo árbol de $n$ nodos asociado a $G_{n}$ cuya raíz es el vértice $1 \mathrm{y}$ $\mathcal{B}=\left\{A_{j}(n), j=1,2, \ldots, n^{n-2}\right\}$ una colección de todos aquellos árboles de $n$ nodos que se pueden obtener a partir de $G_{n}$ y cuyo vértice raíz es el nodo 1 . Si se considera además $A_{j}(n) \in \mathcal{B}$, se puede definir la trayectoria $T_{\{1, q\}}(n)=\left\{\left\{1, l_{1}\right\},\left\{l_{1}, l_{2}\right\}, \ldots,\left\{l_{p}, q\right\}\right\} \subset A_{j}(n)$ definida desde la raíz del árbol y hasta al vértice $q$ como la secuencia 
de aristas que une los vértices 1 y $q$. Notar que una trayectoria del tipo $T_{\{1, q\}}(n)$ representa el camino directo que sigue la energía desde el nodo fuente hasta el nodo $q$ cuando el nodo se conecta a la red utilizando la $j$-ésima topología disponible.

Si se considera un árbol cualquiera $A_{j}(n) \in \mathcal{B}$ que representa un SD de $n$ nodos, entonces la ENS al $i$-ésimo nodo del sistema se puede reescribir como [9][10]:

$$
\operatorname{ENS}_{i}\left(n, A_{j}(n)\right)=\left(\sum_{l \in T_{\{1, i\}}(n)} U_{l}+\sum_{m \in T_{\{1, i\}}^{r}(n)} U_{m}^{\prime}\right) P_{i}
$$

donde $T_{\{1, i\}}^{r}(n)=\left\{\{p, q\} \in A_{j}(n):\{p, q\} \notin T_{\{1, i\}}(n) \wedge\{p, q\}\right.$

se conectan a $\left.T_{\{1, i\}}(n), p, q \neq 0\right\}$ y representa todos aquellos nodos terminales y/o grupos de nodos terminales, que no pertenecen a $T_{\{1, i\}}(n)$ pero que se conectan a $A_{j}(\mathrm{n})$ a través de $T_{\{1, i\}}(n)$ por cualquiera de sus nodos excepto la raíz.

La red radial representada por $A_{j}(\mathrm{n})$ tiene entonces una ENS total, $\operatorname{ENS}\left(n, A_{j}(n)\right)$, dada por:

$$
\operatorname{ENS}\left(n, A_{j}(n)\right) \equiv \operatorname{ENS}(n)=\sum_{i=\{1,2, \ldots, n\}} \operatorname{ENS}_{i}\left(n, A_{j}(n)\right)
$$

Utilizando este nuevo modelo matemático se puede atacar el problema de minimizar la ENS por el SD a través del problema de teoría de grafos de la búsqueda del AME asociado al grafo del SD. Dentro de la literatura asociada a teoría de grafos existen algunos algoritmos apropiados para realizar este tipo de búsquedas, siendo el algoritmo de Prim uno de ellos [14-16]. La principal ventaja de este algoritmo es que su tiempo de ejecución es de orden logarítmico, $O(n, \log (n))$, con respecto al número total de vértices del grafo, lo que le hace ser un algoritmo de búsqueda del tipo voraz. La idea básica del algoritmo de Prim es, comenzando con un árbol con un solo vértice, añadir en cada iteración aquella arista que agregue la menor distancia al árbol. Por lo tanto, para utilizar el algoritmo se debe encontrar primero una expresión recursiva para el cálculo de la ENS. Notar que en el problema de minimizar la ENS, la distancia asociada a cada arista del grafo (ENS) puede variar cuando la topología del SD cambia.
Asúmase que se inicia el algoritmo de Prim con el árbol $\mathrm{A}(1)=\{1\}$, y después de $k-2$ iteraciones se ha obtenido el árbol $A_{j}(k-1)$, formado por el conjunto de vértices $C=\left\{1, l_{1}, \ldots, l_{k-2}\right\}$, cuya cardinalidad es $|C|=k-1$. Durante el proceso de decisión del algoritmo de Prim se desea agregar a $A_{j}(k-1)$ el nodo $\alpha, \alpha \in\left(N_{n} \backslash C\right)$, formando así $A_{j}(k)$. Si utilizamos las ecuaciones (3) y (4) podemos escribir:

$$
\begin{aligned}
& E N S(k)=\sum_{i \in(C \cup\{\alpha\})} E N S_{i}\left(k, A_{j}(k)\right) \\
= & \sum_{i \in\{C \cup\{\alpha\})}\left(\sum_{l \in T_{\{1, i\}}(k)} U_{l}+\sum_{m \in T_{\{1, i\}}^{r}(k)} U_{m}^{\prime}\right) P_{i} \\
= & \sum_{i \in C}\left(\sum_{l \in T_{\{1, i\}}(k)} U_{l}+\sum_{m \in T_{\{1, i\}}^{r}(k)} U_{m}^{\prime}\right) P_{i}+ \\
& +\left(\sum_{l \in T_{\{1, \alpha\}}(k)} U_{l}+\sum_{m \in T_{\{1, \alpha\}}^{r}(k)} U_{m}^{\prime}\right) P_{\alpha}
\end{aligned}
$$

$$
\operatorname{ENS}(k)=\sum_{i \in C}\left(\sum_{l \in T_{\{1, i\}}(k)} U_{l}+\sum_{m \in T_{\{1, i\}}^{r}(k)} U_{m}^{\prime}\right) P_{i}+E N S_{\alpha}\left(k, A_{j}(k)\right)
$$

Para obtener una expresión recursiva podemos separar $A_{j}(k)$ en dos particiones: una que contiene sólo el nodo que está siendo evaluado, $\alpha$, y la otra con los restantes $k-1$ nodos, que corresponden exactamente a $\operatorname{los}(k-1)$ nodos de la etapa anterior. Se debe notar que como $A_{j}(k)$ es un árbol, el nodo $\alpha$ se conecta a él a través de un solo nodo, digamos el nodo $l_{p} \in A_{j}(k-1)$, y la arista $\left\{l_{p}, \alpha\right\}$. De esta forma obtenemos:

$$
\begin{gathered}
E N S(k-1)^{\prime}=\sum_{i \in \mathcal{C}}\left(\sum_{l \in T_{\{1, i\}}(k)} U_{l}+\sum_{m \in T_{\{1, i\}}^{r}(k)} U_{m}^{\prime}\right) P_{i} \\
=\sum_{i \in C}\left(\sum_{l \in T_{\{1, i\}}(k-1)} U_{l}+\sum_{m \in T_{\{1, i\}}^{r}(k-1)} U_{m}^{\prime}\right) P_{i}+ \\
+\sum_{i \in \mathcal{C}} U_{\left\{l_{p}, \alpha\right\}}^{\prime} P_{i} I_{\{i \in \mathcal{R}\}}
\end{gathered}
$$




$$
=\operatorname{ENS}(k-1)+\sum_{i \in \mathcal{C}} U_{\left\{l_{p}, \alpha\right\}}^{\prime} P_{i} I_{\{i \in \mathcal{R}\}}
$$

donde $I_{\{i \in \mathcal{R}\}}$ es la función característica del conjunto $\mathcal{R}$, y está definida como:

$$
I_{\{i \in \mathcal{R}\}}=\left\{\begin{array}{l}
0, i \notin \mathcal{R}=\left(T_{\{1, \alpha\}}(k) \cup T_{\{1, \alpha\}}^{r}(k)\right) \\
1, i \in \mathcal{R}=\left(T_{\{1, \alpha\}}(k) \bigcup T_{\{1, \alpha\}}^{r}(k)\right)
\end{array}\right.
$$

Finalmente, reemplazando (11) en (8) obtenemos una expresión recursiva para el cálculo de la ENS:

$$
\begin{aligned}
E N S(k)= & E N S(k-1)+E N S_{\alpha}\left(k, A_{j}(k)\right)+ \\
& +\sum_{i \in C} U_{\left\{l_{p}, \alpha\right\}}^{\prime} P_{i} I_{\{i \in \mathcal{R}\}}
\end{aligned}
$$

En la ecuación (13) se puede observar que el término $\sum_{i \in C} U_{\left\{l_{p}, \alpha\right\}}^{\prime} P_{i} I_{\{i \in \mathcal{R}\}}$ corresponde a la variación en la ENS de la iteración anterior, producto de la incorporación de un nuevo nodo al SD. Es precisamente este efecto el que hace que el proceso de decisión sea más difícil de evaluar.

En resumen, el algoritmo de Prim busca minimizar la ENS de una red de distribución, con respecto a todas las posibles topologías radiales que se pueden construir usando como nodo fuente el nodo 1 , y sujeto a la restricción de que todos los nodos deben permanecer conectados a la red.

\section{Algoritmo propuesto}

Una vez formulado el problema de optimización para cada etapa del algoritmo, se propone una modificación que permite compensar los efectos de la variabilidad de la métrica de distancia (ENS), lo que afecta las decisiones anteriormente tomadas por el algoritmo. Es decir, debido a que la ENS de un nodo puede cambiar a medida que agregamos nodos al SD, las decisiones óptimas tomadas en la etapa $k$ pueden no seguir siéndolo en las etapas siguientes.

Revisión de la topología resultante: dado que los cambios topológicos pueden producir una modificación en la ENS calculada en las etapas anteriores, al igual que McDecmontt et al. [1], se considera la implementación de una etapa de revisión del AME encontrado por el algoritmo de Prim. Este proceso consiste en almacenar aquellas conexiones que fueron descartadas aleatoriamente (debido a que más de una opción presentaba un camino óptimo para el AME) durante el proceso de decisión. Estas topologías se evalúan empleando nuevamente el algoritmo de Prim a partir de la etapa en la que fueron descartadas. Por lo tanto, la etapa de revisión simplemente continúa el algoritmo de Prim cambiando la decisión que anteriormente fue descartada. Así, se obtienen nuevas topologías que posteriormente se comparan con el AME encontrado inicialmente, obteniendo de esta forma el AME del sistema. Este proceso de revisión tiene dos objetivos: (i) mitigar el efecto de la búsqueda voraz del algoritmo de Prim [1], y (ii) compensar los efectos de la posible variación de la ENS de los nodos cuando un nuevo nodo se agrega a la red.

Algoritmo: A continuación se presenta un resumen de los pasos que el algoritmo propuesto debe seguir para encontrar el AME que representa la RD de mínima ENS:

1. Algoritmo de Prim: Iniciar algoritmo sólo con el nodo 1 asociado al árbol.

2. Considerar todos aquellos nodos directamente conectados al árbol.

a. Evaluar la ENS del árbol más el nodo evaluado usando (13).

b. De entre todos los nodos evaluados, agregar al árbol aquel nodo con menor ENS. En caso de que más de un nodo obtenga la mínima ENS, seleccionar uno aleatoriamente y almacenar las variables asociadas a las otras topologías óptimas.

c. Incrementar en una unidad el contador de nodos que pertenecen al AME.

3. Si el número de nodos del árbol es menor que $n$ volver al paso 2 hasta conectar todos los nodos del SD.

4. Revisión: Evaluar la ENS de las topologías que fueron descartadas aleatoriamente durante el algoritmo de Prim.

\section{EVALUACIÓN TEÓRICA DEL ALGORITMO}

Con el fin de evaluar el desempeño del algoritmo, se consideró el sistema de pruebas mostrado en la figura 2. Los resultados obtenidos al utilizar el algoritmo propuesto se muestran en la tabla 1. El SD a evaluar consta de ocho nodos y ocho líneas de distribución. De la figura 2 se observa que cuatro nodos corresponden a nodos de carga (terminales), tres a nodos de distribución (alimentadores) y el nodo 1 corresponde al nodo fuente. Inicialmente se considera que las ocho líneas poseen interruptores, por lo tanto, cualquier topología radial puede ser formada a 
partir del SD. También de la figura 2 pueden obtenerse los parámetros de confiabilidad del sistema, es decir, las indisponibilidades asociadas al tiempo de restauración y al tiempo de reparación y las potencias activas de cada nodo.

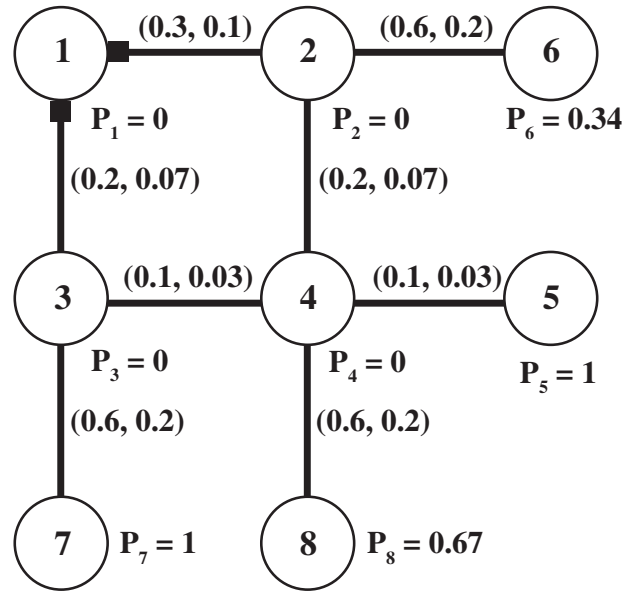

Figura 2. Sistema de pruebas formado por ocho nodos y ocho líneas de distribución de energía. Los valores de potencia activa de cada nodo son denotados como $P_{i}$ (en $\left.[\mathrm{KW}]\right)$, mientras que el par ordenado $\left(U, U^{\prime}\right)$ representa las indisponibilidades asociadas a los tiempos de reparación y restauración de cada línea (en [h/año]), respectivamente.

Tabla 1. Resultados obtenidos tras la aplicación del algoritmo propuesto al SD de la figura 2. El símbolo $†$ representa los valores calculados por el algoritmo antes de la etapa de revisión, mientras que $\$$ corresponde a los valores finales entregados por el algoritmo.

\begin{tabular}{|l|c|}
\hline Iteraciones & 7 \\
\hline Línea Abierta $\uparrow$ & $\{3,4\}$ \\
\hline ENS [KWh/año] $\dagger$ & 3.099 \\
\hline Línea Abierta + & $\{2,4\}$ \\
\hline ENS [KWh/año] $\dagger$ & 2.923 \\
\hline
\end{tabular}

Se procedió a ejecutar el algoritmo propuesto, obteniéndose una ENS de 3.099 [KWh/año] una vez finalizado el algoritmo de Prim, y una vez ejecutada la etapa de revisión se encontró una topología alternativa, abriendo la línea $\{2,4\}$, que posee una ENS de 2.923 [KWh/año]. De la figura 2 se puede observar claramente que existen sólo cuatro topologías radiales, a las que se les calculó su respectiva ENS, y los resultados obtenidos se listan en la tabla 2. Se puede observar que para el sistema de pruebas de la figura 2, la topología de mínima ENS se produce al abrir la línea $\{2,4\}$, topología que concuerda con la obtenida por el algoritmo propuesto. Finalmente, es fácil ver que la cantidad de iteraciones ejecutadas por el algoritmo de Prim es un valor conocido: $n-1$.

Tabla 2. Resultados obtenidos para cada una de las opciones de radialidad del SD de la figura 2 cuando todas las líneas tienen interruptor.

\begin{tabular}{|l|c|c|c|c|}
\hline Línea Abierta & $\{1,2\}$ & $\{2,4\}$ & $\{1,3\}$ & $\{3,4\}$ \\
\hline ENS [KW/año] & 3.858 & 2.923 & 4.391 & 3.099 \\
\hline
\end{tabular}

\section{OPTIMIZACIÓN DE REDES REALES}

A continuación se muestran los resultados obtenidos al aplicar el método propuesto a algunos SD reales. En la figura 3 se puede observar un SD de cuarenta nodos, dos fuentes de alimentación, cuarenta y una líneas de distribución y diez nodos de carga (que están rotulados con un círculo). Los parámetros de confiabilidad asociados al SD de la figura se listan en la tabla 4 del apéndice.

Inicialmente se estudiará el SD dividiéndolo en dos subsistemas mediante la apertura de la línea $\{40,41\}$. Con esto se forman los llamados sistema izquierdo y sistema derecho, cada uno con un nodo fuente (nodos 28 y 38 , respectivamente), veinte líneas de distribución y diecinueve nodos: nueve nodos de carga, donde $P_{i}>0$, y diez nodos de distribución cuya potencia activa es cero, es decir, $P_{i}=0$. Notar que debido a la existencia de dos fuentes de alimentación, el algoritmo no es directamente aplicable, por lo que se utilizará una modificación al SD original que consiste en: (i) crear una fuente de alimentación ficticia, que pasará a ser el nodo fuente, y (ii) agregar dos líneas de distribución ideales (es decir, con indisponibilidades nulas) que conectan el nuevo nodo fuente a los nodos 28 y 38. De esta forma se puede analizar el SD de la figura 3 utilizando este SD equivalente que posee cuarenta y un nodos y cuarenta y tres líneas de distribución.

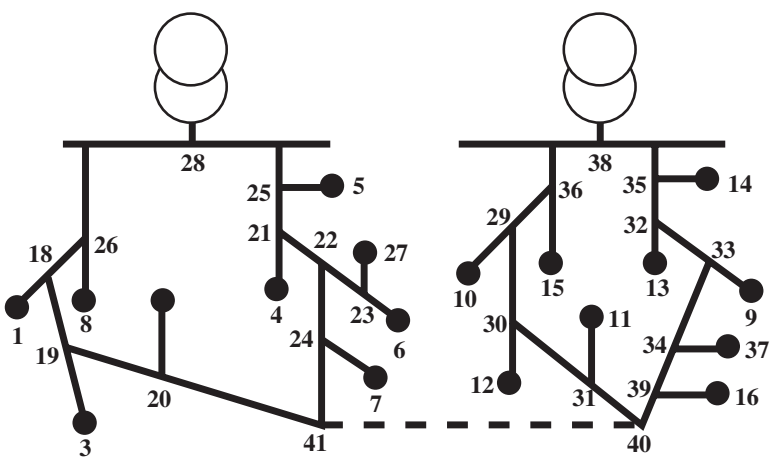

Figura 3. SD real formado por cuarenta nodos y cuarenta y una líneas de distribución. 
Los resultados de la evaluación de los sistemas izquierdo y derecho, así como del SD completo, se listan en la tabla 3. Con fines de comparación, en la misma tabla también se muestran los valores de ENS óptima, ENS*, para cada sistema. Estos valores fueron obtenidos por medio de la búsqueda exhaustiva de la(s) topología(s) óptima(s) del sistema. A la luz de los resultados obtenidos se puede observar que el algoritmo propuesto es capaz de obtener una topología radial con mínima ENS ejecutando una búsqueda considerablemente más eficiente que la desarrollada por un método exhaustivo. En el caso del SD completo se observa que el algoritmo propuesto no obtiene la topología óptima que se encontró vía búsqueda exhaustiva, pues ésta se produce al abrir las líneas: $\{19,20\}$, $\{31,40\}$ y $\{39,40\}$. A pesar de que la solución entregada por el algoritmo es subóptima, en aplicaciones como SD reales donde existe una enorme cantidad de nodos conectados al sistema, obtener una topología cercana a la óptima es una solución bastante apetecida.

Tabla 3. Resultados obtenidos tras la aplicación del algoritmo propuesto al SD de la figura 3.

\begin{tabular}{|l|c|c|}
\hline Sist. Izquierdo & Prerrevisión & Postrevisión \\
\hline ENS [KWh/año] & 6016 & 5956 \\
\hline Línea abierta & $\{24,41\}$ & $\{20,41\}$ \\
\hline ENS* [KWh/año] & 5956 & 5956 \\
\hline Sist. Derecho & Prerrevisión & Postrevisión \\
\hline ENS [KWh/año] & 5310 & 5310 \\
\hline Línea abierta & $\{31,40\}$ & $\{31,40\}$ \\
\hline ENS* [KWh/año] & 5310 & 5310 \\
\hline Sist. Completo & Prerrevisión & Postrevisión \\
\hline ENS [KWh/año] & 19808 & 19359 \\
\hline Líneas abiertas & $\{24,41\}$, & $\{20,41\}$, \\
\hline ENS* [KWh/año] & $190,41\},\{39,40\}$ & $\{31,40\},\{39,40\}$ \\
\hline \hline Sist. CGE & Prerrevisión & Postrevisión \\
\hline ENS [MWh/año] & 130.33 & 130.33 \\
\hline
\end{tabular}

Adicionalmente, se procedió a evaluar un segundo SD real, llamado sistema CGE, que está formado por novecientas cincuenta y siete líneas de distribución y novecientos diecisiete nodos, de los cuales uno corresponde a una fuente de distribución y cuatrocientos sesenta y cuatro son nodos de carga. Los resultados obtenidos para este sistema se listan en la tabla 3.

\section{CONCLUSIONES}

En este trabajo se introduce un nuevo algoritmo que permite optimizar sistemas de distribución de media tensión minimizando la energía no suministrada del sistema. Para derivar el algoritmo, se ha desarrollado una rigurosa modelación matemática de los sistemas de distribución basada en teoría de grafos. Esta modelación ha permitido además derivar una expresión iterativa para el cálculo de la ENS, la que es utilizada en conjunto con el algoritmo de Prim para producir un algoritmo de búsqueda de árboles de mínima expansión que resulta ser muy eficiente. Específicamente, el algoritmo propuesto encuentra una topología radial en tal sólo $n-1$ iteraciones, donde $n$ es el número de nodos del sistema de distribución. Es esta eficiencia de cálculo la que hace que el algoritmo propuesto sea bastante atractivo para analizar sistemas de distribución reales que tienen una gran cantidad de nodos. Además, tanto la modelación como la metodología propuesta en este trabajo resultan ser fácilmente extensibles a otros funcionales de optimización utilizados en el análisis de redes tanto eléctricos como de telecomunicaciones, entre otras aplicaciones.

El algoritmo desarrollado considera también la ejecución de una etapa de revisión de la topología radial generada, lo que permite compensar: (i) los efectos de la toma de decisiones que en una cierta iteración fueron óptimas, pero que producto de la variación de la topología no resultan serlo en decisiones futuras, y (ii) los efectos de decisiones aleatorias tomadas en alguna iteración producto de encontrar más de una topología de mínima ENS.

\section{AGRADECIMIENTOS}

Este trabajo se realizó con el financiamiento de la Dirección de Investigación de la Universidad de Concepción, Chile en el proyecto DIUC 204.92.44-1. Además, los autores desean agradecer a la Sra. Flor Espinoza Hidalgo, del Department of Mathematics and Statistics, The University of New Mexico, Albuquerque, NM, USA y a los Srs. Sebastián Godoy Medel y Cristian Sepúlveda Villegas de la Universidad de Concepción, Concepción, Chile, por sus valiosos comentarios y sugerencias durante la elaboración de este trabajo.

\section{APÉNDICE}

En la tabla 4 se listan los parámetros de confiabilidad (tasa de falla, tiempo de reparación y tiempo de restauración de cada línea) asociados al SD de la figura 3. 
Tabla 4. Parámetros de confiabilidad del SD de la figura 3.

\begin{tabular}{|c|c|c|c|c|}
\hline $\begin{array}{c}\text { Línea } \\
\{l, m\}\end{array}$ & $\lambda_{\{l, m\}}[1 /$ año $]$ & $t_{\{l, m\}}^{(r e p)}[\mathrm{h}]$ & $t_{\{l, m\}}^{(r e s t)}[\mathrm{h}]$ & $P_{m}[\mathrm{KW}]$ \\
\hline$\{18,1\}$ & 0.25 & 2.50 & 1.25 & 400 \\
\hline$\{18,19\}$ & 0.20 & 1.50 & 0.75 & 0 \\
\hline$\{19,3\}$ & 0.15 & 2.50 & 1.25 & 400 \\
\hline$\{19,20\}$ & 0.20 & 1.50 & 0.75 & 0 \\
\hline$\{20,2\}$ & 0.15 & 2.50 & 1.25 & 500 \\
\hline$\{20,41\}$ & 0.20 & 1.50 & 0.75 & 0 \\
\hline$\{21,4\}$ & 0.15 & 2.50 & 1.25 & 150 \\
\hline$\{21,22\}$ & 0.20 & 1.50 & 0.75 & 0 \\
\hline$\{22,23\}$ & 0.15 & 2.50 & 1.25 & 0 \\
\hline$\{22,24\}$ & 0.20 & 1.50 & 0.75 & 0 \\
\hline$\{23,6\}$ & 0.15 & 2.50 & 1.25 & 200 \\
\hline$\{23,27\}$ & 0.15 & 2.50 & 1.25 & 200 \\
\hline$\{24,7\}$ & 0.15 & 2.50 & 1.25 & 150 \\
\hline$\{24,41\}$ & 0.20 & 1.50 & 0.75 & 0 \\
\hline$\{25,5\}$ & 0.15 & 2.50 & 1.25 & 500 \\
\hline$\{25,21\}$ & 0.10 & 1.50 & 0.75 & 0 \\
\hline$\{26,8\}$ & 0.15 & 2.50 & 1.25 & 300 \\
\hline$\{26,18\}$ & 0.25 & 2.50 & 0.75 & 0 \\
\hline$\{28,25\}$ & 0.05 & 1.50 & 0.75 & 0 \\
\hline$\{28,26\}$ & 0.05 & 1.50 & 0.75 & 0 \\
\hline$\{29,10\}$ & 0.15 & 2.50 & 1.25 & 500 \\
\hline$\{29,30\}$ & 0.20 & 1.50 & 0.75 & 0 \\
\hline$\{30,12\}$ & 0.20 & 1.50 & 0.75 & 400 \\
\hline$\{30,31\}$ & 0.20 & 1.50 & 0.75 & 0 \\
\hline$\{31,11\}$ & 0.15 & 2.50 & 1.25 & 300 \\
\hline$\{31,40\}$ & 0.20 & 1.50 & 0.75 & 0 \\
\hline$\{32,13\}$ & 0.15 & 2.50 & 1.25 & 300 \\
\hline$\{32,33\}$ & 0.20 & 1.50 & 0.75 & 0 \\
\hline$\{33,9\}$ & 0.15 & 2.50 & 1.25 & 400 \\
\hline$\{33,34\}$ & 0.20 & 1.50 & 0.75 & 0 \\
\hline$\{34,37\}$ & 0.15 & 2.50 & 1.25 & 150 \\
\hline$\{34,39\}$ & 0.20 & 1.50 & 0.75 & 0 \\
\hline$\{35,14\}$ & 0.15 & 2.50 & 1.25 & 500 \\
\hline$\{35,32\}$ & 0.10 & 1.50 & 0.75 & 0 \\
\hline$\{36,15\}$ & 0.15 & 2.50 & 1.25 & 300 \\
\hline$\{36,29\}$ & 0.10 & 1.50 & 0.75 & 0 \\
\hline$\{38,35\}$ & 0.05 & 1.50 & 0.75 & 0 \\
\hline$\{38,36\}$ & 0.05 & 1.50 & 0.75 & 0 \\
\hline$\{39,16\}$ & 0.15 & 2.50 & 1.25 & 150 \\
\hline$\{39,40\}$ & 0.20 & 1.50 & 0.75 & 0 \\
\hline$\{40,41\}$ & 0.00 & 0.00 & 0.00 & 0 \\
\hline
\end{tabular}

\section{REFERENCIAS}

[1] Reglamento de la Ley General de Servicios Eléctricos. Decreto Supremo 327. República de Chile. 1998.

[2] T. McDermott, I. Drezga y R. Broadwater. "A heuristic nonlinear constructive method for distribution system reconfiguration". IEEE Trans. on Power System. Vol. $14 \mathrm{~N}^{\mathrm{o}}$ 2, pp. 478-483. Mayo 1999.
[3] S.K. Goswami y S.K. Basu. "A new algorithm for the reconfiguration of distribution feeders for loss minimization". IEEE Trans. on Power Delivery. Vol. $7 \mathrm{~N}^{\circ}$ 3, pp. 1484-1491. Julio 1992.

[4] F.V. Gomes, S. Carneiro Jr., J.L.R. Pereira, M.P. Vinagre, P.A.N. Garcia y L.R. Araujo. "A new heuristic reconfiguration algorithm for large distribution systems". IEEE Trans. on Power Systems. Vol. $20 \mathrm{~N}^{\circ}$ 3, pp. 1373-1378. Agosto 2005.

[5] D. Shirmohammadi y H.W. Hong. "Reconfiguration of electric distribution networks for resistive line losses reduction". IEEE Trans. on Power Delivery. Vol. $4 \mathrm{~N}^{\circ}$ 2, pp. 1492-1498. Abril 1989.

[6] S. Toune, H. Fudo, T. Genji, Y. Fukuyama y Y. Nakanishi, "Comparative study of modern heuristic algorithms to service restoration in distribution systems". IEEE Trans. on Power Delivery. Vol.17 No 1, pp. 173-181. Enero 2002.

[7] E. López, H. Opazo, P. Pedrero, S. Quiroz. "Optimización de pérdidas en redes de distribución". XI Congreso de la Asociación Chilena de Control Automático. Concepción, Chile. 1994.

[8] E. López, H. Opazo, L. García, M. Poloujadoff. "Minimal loss reconfiguration based on dynamic programming approach: Application to real system". Electric Power Component and System. Vol. 30, pp. 693-704. Julio 2002.

[9] W. Min, H. Chan. "A new approach for distribution feeder reconfiguration for loss reduction and service restoration". IEEE Trans. on Power Delivery. Vol. 13, pp. 870-875. Julio 1998.

[10] M. Vega, H. Sarmiento, L. Matías. "Reconfiguring a primary industrial network based on reliability studies". Industrial Applications Conference. Vol. 2, pp. 1095-1099. Octubre 2002.

[11] D. Popovic, R. Ciric. "A multi-objective algorithm for distribution network restoration". IEEE Trans. on Power Delivery. Vol. 14, pp. 1134-1141. Julio 1999.

[12] D. Popovic, Z. Popovic. "A risk management procedure for supply restoration in distribution networks". IEEE Trans. on Power System. Vol. 19, pp. 221-228. Febrero 2004. 
[13] C. Huang. "Multiobjective service restauration of distribution system using fuzzy cause effect network". IEEE Trans. on Power System. Vol. 18, pp. 867-874. Mayo 2003.

[14] J.R.A. Rodríguez y A. Vargas. "Fuzzy-heuristic methodology to estimate the load restoration time in MV networks". IEEE. Trans. on Power Systems. Vol. $20 \mathrm{~N}^{\circ}$ 2, pp. 1095-1102. Mayo 2005.
[15] T. H. Cormen, C. E. Leiserson, R. L. Rivest, C. Stein. "Introduction to algorithms". Segunda Edición. The MIT Press. 2001.

[16] R. Diestel. "Graph theory". Tercera Edición. Springer-Verlag. Heidelberg. 2005.

[17] R.C. Prim. "Shortest connection networks and some generalisations". Bell System Technical Journal. Vol. 36, pp. 1389-1401. 1957. 